\title{
Intensional Models for the Theory of Types*
}

\author{
Reinhard Muskens
}

\begin{abstract}
In this paper we define intensional models for the classical theory of types, thus arriving at an intensional type logic ITL. Intensional models generalize Henkin's general models and have a natural definition. As a class they do not validate the axiom of Extensionality. We give a cut-free sequent calculus for type theory and show completeness of this calculus with respect to the class of intensional models via a model existence theorem. After this we turn our attention to applications. Firstly, it is argued that, since ITL is truly intensional, it can be used to model ascriptions of propositional attitude without predicting logical omniscience. In order to illustrate this a small fragment of English is defined and provided with an ITL semantics. Secondly, it is shown that ITL models contain certain objects that can be identified with possible worlds. Essential elements of modal logic become available within classical type theory once the axiom of Extensionality is given up.
\end{abstract}

\section{Introduction}

The axiom scheme of Extensionality states that whenever two predicates or relations are coextensive they must have the same properties:

$$
\forall X Y(\forall \vec{x}(X \vec{x} \leftrightarrow Y \vec{x}) \rightarrow \forall Z(Z X \rightarrow Z Y))
$$

Historically Extensionality has always been problematic, the main problem being that in many areas of application, though not perhaps in the foundations of mathematics, the statement is simply false. This was recognized by Whitehead and Russell in Principia Mathematica [32, where intensional functions such as ' $A$ believes that $p$ ' or 'it is a strange coincidence that $p$ ' are discussed at length. However, in the introduction to the second edition (1927) of the Principia Whitehead and Russell (influenced by Wittgenstein's Tractatus) already entertain the possibility that "all functions of functions are extensional". Thirteen years later, in Church's [ 6 canonical formulation of the Theory of Types, it is observed that axioms of Extensionality should be adopted "[i]n order to obtain classical real number theory (analysis)", a wording that does not seem to rule out the option of not adopting them. Church's formulation of type theory was completely syntactic and axioms could be adopted or dropped at will,

*The Journal of Symbolic Logic, to appear. 
but in Henkin's 12] classical proof of generalized completeness the models that are considered, both the "standard" models and the "general" ones, simply validate Extensionality. Although Henkin's text still allows giving up the axiom ${ }^{1}$ the formal set-up now effectively rules out intensional predicates and functions.

This poses problems for those areas of application of the logic where it is important to distinguish between predicates that are coextensive and where propositions that determine the same set of possible worlds should be kept apart nevertheless. Linguistic semantics and Artificial Intelligence are such applications and the problem has been dubbed one of "logical omniscience" there, for it is with propositional attitudes like knowledge and belief that predicates of predicates and predicates of propositions most naturally arise. Is there a deep foundational difficulty with type theory that makes the theory adequate for one area of application (mathematics) but not for others? Or is it possible to come up with a revised and generalized semantics for the logic, in which intensional predicates of predicates (or intensional functions of functions) are allowed? In the latter case Extensionality becomes a non-logical axiom that can be added to the theory for the purposes of one area of application while in other areas of application it is not added.

Even if one is interested in mathematical applications of type theory only there are good reasons to consider a generalization of its models in which Extensionality fails. This was realized by Takahashi 28] and Prawitz 24] in their (independent, but closely related) proofs of Cut-elimination. These proofs make use of what Andrews [1] calls " $V$-complexes", structures whose typed domains consist of elements $\langle A, e\rangle$, where $A$ is a term and $e$ is a possible extension of $A$. Clearly, two objects $\langle A, e\rangle$ and $\left\langle B, e^{\prime}\right\rangle$ can be distinct even if $e=e^{\prime}$. Andrews [1] uses $V$-complexes to show that a certain resolution system $\mathcal{R}$ corresponds to the first six axioms of Church [ [ (not comprising Extensionality).

$V$-complexes in themselves cannot be used as independent models for an intensional type theory, as their definition depends on Schütte's [26] "semivaluations", essentially sets of sentences (the " $V$ " in " $V$-complex" ranges over semi-valuations). Is it possible to define a stand-alone notion of general intensional model that has $V$-complexes as a special case? I know of two proposals for such general models, both recent. The first is found in Fitting [10, the second in Benzmüller et al. [3]. In Fitting's "generalized Henkin models" abstraction may receive a non-standard interpretation, while in the " $\Sigma$-models" of Benzmüller et al. it is application that may be interpreted in a non-standard way. Such non-standard evaluations seem unnecessary, however, and in this paper, I will propose a simple definition of intensional model that generalizes Henkin models for type theory but gives all logical operations their usual semantics. The system of type theory interpreted with the help of these intensional models will be called ITL ('Intensional Type Logic').

The rest of the paper is organized as follows. In the following section we will consider some existing proposals to obtain intensionality and we will argue

\footnotetext{
${ }^{1}$ Henkin 12: The axioms of extensionality ... can be dropped if we are willing to admit models whose domains contain functions which are regarded as distinct even though they have the same value for every argument.
} 
that they all have a simple pattern in common that can be used to obtain a general intensional logic. Section 3 gives the types and terms of a type theory in the spirit of Church [6] (but framed as a relational theory, as in Orey [22] and Schütte [26]). In section [ 1 our notion of intensional model will be defined, with a corresponding notion of entailment. Section $[5$ introduces a cut-free Gentzen calculus for ITL while section [ proves a Model Existence theorem. The proofs in that section all employ familiar techniques but are given as a sanity check on the definition of the basic modeltheoretic notions. The last two sections consider applications: Section [7 uses the logic to provide a fragment of natural language with a truly intensional semantics while section 8 shows how possible worlds can be obtained as certain objects in intensional models. A short conclusion ends the paper.

\section{Informal Analysis}

Given Leibniz's principle of the identity of indistinguishables and the assumption that $\forall$ and $\rightarrow$ behave classically, $\forall Z(Z X \rightarrow Z Y)$ implies $X=Y$ and

$$
\forall X Y(\forall \vec{x}(X \vec{x} \leftrightarrow Y \vec{x}) \rightarrow X=Y)
$$

therefore will be equivalent with Extensionality. This means that a semantics in which this axiom fails cannot under reasonable assumptions identify the semantic value of an expression with its extension, as $\forall \vec{x}(X \vec{x} \leftrightarrow Y \vec{x})$ just states that $X$ and $Y$ are co-extensive. The following is a propositional instantiation of (2) (with the length of $\vec{x}$ set to 0 and $X$ and $Y$ instantiated as $\varphi$ and $\psi$ respectively).

$$
(\varphi \leftrightarrow \psi) \rightarrow \varphi=\psi
$$

Here $\varphi \leftrightarrow \psi$ expresses that $\varphi$ and $\psi$ have the same truth value whereas $\varphi=\psi$ says they are the same proposition. Typically we want this scheme to fail, as sentences with the same truth values may be distinguishable in the sense that one is believed while the other is not, or that one is a strange coincidence, while the other is entirely expected, etc. Many propositions must therefore be allowed to exist, although each proposition must assume one of two truth values if we want to retain classicality.

Although the semantic values of sentences cannot be equated with their truth values, it still seems reasonable to require that they should determine these truth values, while the values of expressions of higher type should likewise determine their extensions. If this is accepted the picture that arises is that logical expressions are sent to their (intensional) values by some function $I$, while these values in their turn are connected with extensions by a function $E$. The latter typically does not need to be injective. Readers familiar with the extensive literature on intensionality (sometimes dubbed hyperintensionality) will be aware that, while there are many divergent proposals for what intensions are, the pattern just sketched is well-nigh ubiquitous. Already in Frege's [1] pivotal work an expression expresses a sense (the function $I$ ) while a sense in its 
turn determines a reference $(E)$. In modal logic, intensions are functions from possible worlds to extensions (let us call such functions modal intensions) and the function $E$ can be viewed as application of such functions to a fixed 'actual' world $w_{0}$. It is obvious that this modal strategy does not individuate intensions finely enough (essentially since, if $W_{1}$ and $W_{2}$ denote sets of possible worlds or the characteristic functions of such sets, $\forall w\left(W_{1} w \leftrightarrow W_{2} w\right) \rightarrow W_{1}=W_{2}$ will hold) and many researchers have sought notions of intension coming with more fine-grained criteria of individuation than modal intensions come with. Carnap 4, for example, who defined a precursor to the now usual possible worlds analysis of natural language, noticed the problems that this analysis suffers from and proposed a theory of structured meanings that was later worked out in Lewis 14 and Cresswell [8]. On Lewis' account the meaning of an expression is a finite ordered tree having at each node a category and an appropriate modal intension. The modal intension at the root node of this tree is the one associated with the expression as a whole and so in this theory the function $I$ assigns finite ordered trees of categories and modal intensions to any given expression, while $E$ is the function that takes any such tree and returns $W w_{0}$, where $W$ is the modal intension found at its root node.

Another example of an approach in which the functions $I$ and $E$ can easily be recognised is the theory of impossible worlds. The idea behind this line of thought is that if the usual set of possible worlds is not large enough to make enough distinctions between semantic values, extra worlds, impossible ones, should be added. A key point is that the logical operators need not have their usual meaning at these points of reference and that logical validities will therefore cease to hold throughout the set of all worlds. The name "impossible (possible) world" derives from Hintikka [13, but the idea was also present in Montague [15] and Cresswell [7] and has been followed up in Rantala [25, Barwise [2], and Zalta 34, for example. The function $I$ here is the function that sends each sentence to a set of possible and impossible worlds, wheras $E$ can be described as $\lambda W . W w_{0}$, as in the ordinary modal account.

Other approaches to intensionality may have different conceptions of the nature of intensions, but will also follow a two-stage pattern in which expressions are first sent to their intensions (whatever these are) and intensions are subsequently related to extensions. For example, in Property Theory (Turner 31, Chierchia and Turner 5 ) one finds a homomorphism $T$ sending an algebra of 'information units' $\mathbf{I}$ to a boolean algebra $\mathbf{P}$ (Chierchia and Turner [5]). Here the information units act as intensions while the elements of $\mathbf{P}$ are the extensions and $T$ is the function we have called $E$. Thomason [30 uses a higher-order logic that obeys a form of Extensionality to interpret natural language sentences in a domain $D_{p}$ of propositions considered as primitive entities and then uses a function ${ }^{\cup}$ (our $E$ ) to send these propositions to their extensions (see also Muskens [20]). Moschovakis [17, to give a last example, identifies senses with algorithms and references with the values that these algorithms return. Here the function that sends expressions to algorithms is our $I$ while $E$ assigns to each algorithm the value returned.

Thus while opinions about the nature of intensions radically diverge, all pro- 
posals follow a simple two-stage pattern. The aim of this paper is not to add one more theory of intension to the proposals that have already been made, but is an investigation of their common underlying logic. The idea will be that the two-stage set-up is essentially all that is needed to obtain intensionality. For the purposes of logic it suffices to consider intensions as abstract objects; the question what intensions are, while philosophically important, can be abstracted from. Conversely, while many positions regarding the ultimate nature of intensions seem rationally possible and no knock-down arguments are likely to decide the matter, it does equally seem possible to rationally converge on a logic describing what intensions $d o$. Here we attempt to contribute to that logic.

\section{Terms}

In this section the types and terms of ITL will be defined and some notation will be adopted. While this ITL syntax will be given an intensional interpretation in the next section, it essentially is the syntax of the simple type theory of Church's [6]. The intended interpretation will be relational, however, as in Orey 22] and Schütte [26], not functional, as in Church's original work.

Assuming that some finite set $\mathcal{B}$ of basic types is given, the following definition gives the set of types.

Definition 1. The set $\mathcal{T}$ of types is the smallest set of strings such that

1. $\mathcal{B} \subseteq \mathcal{T}$

2. If $\alpha_{1}, \ldots, \alpha_{n} \in \mathcal{T}(n \geq 0)$ then $\left\langle\alpha_{1} \ldots \alpha_{n}\right\rangle \in \mathcal{T}$

Types formed with the second clause of this definition will be called complex. The intended interpretation is that the extension of an object of type $\left\langle\alpha_{1} \ldots \alpha_{n}\right\rangle$ is an $n$-place relation taking objects of type $\alpha_{i}$ as its $i$-th argument. Note that, as a limiting case, \langle\rangle is defined to be a (complex) type; this will be the type of propositions, with truth values as extensions.

A language will be a countable set of uniquely typed non-logical constants. If $\mathcal{L}$ is a language, the set of constants from $\mathcal{L}$ that have type $\alpha$ is denoted $\mathcal{L}_{\alpha}$. For each $\alpha \in \mathcal{T}$ we moreover assume the existence of a denumerably infinite set $\mathcal{V}_{\alpha}$ of variables with unique type $\alpha$. We let $\mathcal{V}=\bigcup_{\alpha \in \mathcal{T}} \mathcal{V}_{\alpha}$

The following definition gives us terms in all types. Apart from variables and non-logical vocabulary there will be a sentence $\perp$ that is always false, and there will be application and abstraction. Furthermore, a symbol $\subset$ will denote inclusion of extensions, so that $A \subset B$ is true if the extension of $A$ is a subset of that of $B$.

Definition 2. Let $\mathcal{L}$ be a language. Define sets $T_{\alpha}^{\mathcal{L}}$ of terms of $\mathcal{L}$ of type $\alpha$, for each $\alpha \in \mathcal{T}$, as follows.

1. $\mathcal{L}_{\alpha} \subseteq T_{\alpha}^{\mathcal{L}}$ and $\mathcal{V}_{\alpha} \subseteq T_{\alpha}^{\mathcal{L}}$ for each $\alpha \in \mathcal{T}$ 
2. $\perp \in T_{\langle\rangle}^{\mathcal{L}}$

3. If $A \in T_{\left\langle\alpha_{1} \alpha_{2} \ldots \alpha_{n}\right\rangle}^{\mathcal{L}}$ and $B \in T_{\alpha_{1}}^{\mathcal{L}}$, then $(A B) \in T_{\left\langle\alpha_{2} \ldots \alpha_{n}\right\rangle}^{\mathcal{L}}$

4. If $A \in T_{\left\langle\alpha_{2} \ldots \alpha_{n}\right\rangle}^{\mathcal{L}}$ and $x \in \mathcal{V}_{\alpha_{1}}$, then $(\lambda x . A) \in T_{\left\langle\alpha_{1} \alpha_{2} \ldots \alpha_{n}\right\rangle}^{\mathcal{L}}$

5. If $A \in T_{\alpha}^{\mathcal{L}}$ and $B \in T_{\alpha}^{\mathcal{L}}$ then $(A \subset B) \in T_{\langle\rangle}^{\mathcal{L}}$, if $\alpha$ is complex

We will write $T^{\mathcal{L}}$ for the set of all terms of the language $\mathcal{L}$, i.e. for the union $\bigcup_{\alpha \in \mathcal{T}} T_{\alpha}^{\mathcal{L}}$. If $A$ is a term of type $\alpha$, we may indicate this by writing $A_{\alpha}$ and we will use $\varphi, \psi, \chi$ for terms of type \langle\rangle , which we call formulas. The notions free and bound occurrence of a variable and the notion $B$ is free for $x$ in $A$ are defined as usual, as are closed terms and sentences. Substitutions are functions $\sigma$ from variables to terms such that $\sigma(x)$ has the same type as $x$. If $\sigma$ is a substitution then the substitution $\sigma^{\prime}$ such that $\sigma^{\prime}(x)=A$ and $\sigma^{\prime}(y)=\sigma(y)$ for all $y \not \equiv x$ is denoted as $\sigma[x:=A]$. If $A$ is a term and $\sigma$ is a substitution, $A \sigma$, the extension of $\sigma$ to $A$, is defined in the usual way. The substitution $\sigma$ such that $\sigma\left(x_{i}\right)=A_{i}$ and $\sigma(y)=y$ if $y \notin\left\{x_{1}, \ldots, x_{n}\right\}$ is written as $\left\{x_{1}:=A_{1}, \ldots, x_{n}:=A_{n}\right\}$. Parentheses in terms will often be dropped on the understanding that $A B C$ is $((A B) C)$, i.e. association is to the left.

Our stock of operators may seem somewhat spartan, but is rich enough to let the usual connectives and quantifiers be defined. In particular, $\forall, \rightarrow$ and $=$ are easily obtained.

Definition 3. Write

$$
\begin{array}{rll}
\varphi \rightarrow \psi & \text { for } & \varphi \subset \psi, \\
\top & \text { for } & \perp \rightarrow \perp, \\
\forall x \varphi & \text { for } & (\lambda x \cdot \top) \subset(\lambda x \cdot \varphi), \text { and } \\
A_{\alpha}=B_{\alpha} & \text { for } & \forall x_{\langle\alpha\rangle}(x A \rightarrow x B) .
\end{array}
$$

The operators $\neg, \wedge, \vee, \leftrightarrow$ and $\exists$ are defined as usual.

Our presentation of the logic will revolve around sequents. A signed sentence of $\mathcal{L}$ will be a pair $\langle\mathrm{L}, \varphi\rangle$ (written $\mathrm{L}: \varphi$ ) or a pair $\langle\mathrm{R}, \varphi\rangle$ (written $\mathrm{R}: \varphi$ ), such that $\varphi$ is a sentence of $\mathcal{L}$ ( $L$ indicates 'left' and R indicates 'right'). A sequent of $\mathcal{L}$ is a set of signed sentences of $\mathcal{L}$. Letting sequents be sets has some advantages, but we may also want to use a more conventional form and write $\Pi \Rightarrow \Sigma$ for $\{\mathrm{L}: \varphi \mid \varphi \in \Pi\} \cup\{\mathrm{R}: \varphi \mid \varphi \in \Sigma\}$ if $\Pi$ and $\Sigma$ are sets of sentences.

\section{Intensional Models}

Let us turn to the semantics of ITL, which will essentially follow the two-stage pattern discussed above. The following definition sets up the usual hierarchies of objects and provides some of the usual notation.

Definition 4. A collection of domains will be a set $\left\{D_{\alpha} \mid \alpha \in \mathcal{T}\right\}$ of pairwise disjoint non-empty sets. An assignment a for a collection of domains $D=$ 
$\left\{D_{\alpha} \mid \alpha \in \mathcal{T}\right\}$ is a function which has the set of variables $\mathcal{V}$ as domain and has the property that $a(x) \in D_{\alpha}$ if $x \in \mathcal{V}_{\alpha}$. The set of all assignments for $D$ is denoted $\mathcal{A}_{D}$. If $a$ is an assignment, $d \in D_{\alpha}$, and $x$ is a variable of type $\alpha$, $a[d / x]$ is defined by letting $a[d / x](x)=d$ and $a[d / x](y)=a(y)$, if $y$ is not equal to $x$.

Note that we have not imposed any non-trivial relations between the elements of any given collection of domains $D$. In particular we have not required domains $D_{\left\langle\alpha_{1} \ldots \alpha_{n}\right\rangle}$ to consist of relations over lower domains. This is because we need to tease apart the intensions and extensions of terms of complex type. While extensions of such terms will be certain relations, with their identity criteria therefore given by set membership, the intension functions defined below send terms to almost arbitrary domain elements.

Definition 5. An intension function for a collection of domains $D=\left\{D_{\alpha} \mid\right.$ $\alpha \in \mathcal{T}\}$ and a language $\mathcal{L}$ is a function $I: \mathcal{A}_{D} \times T^{\mathcal{L}} \rightarrow D$ such that

1. $I(a, A) \in D_{\alpha}$, if $A$ is of type $\alpha$

2. $I(a, x)=a(x)$, if $x$ is a variable

3. $I(a, A)=I\left(a^{\prime}, A\right)$, if $a$ and $a^{\prime}$ agree on all variables free in $A$

4. $I(a, A\{x:=B\})=I(a[I(a, B) / x], A)$, if $B$ is free for $x$ in $A$

Intension functions are the formal counterpart of the functions $I$ that were discussed informally above. They take an extra assignment argument in order to take care of free variables.

The second part of our formalisation of the two-stage architecture discussed above are the extension functions of definition [6 They send objects of complex types to certain relations over the relevant domains. We first give very general constraints and will put more requirements on useful extension functions in definition 8

Definition 6. An extension function for a collection of domains $D=\left\{D_{\alpha} \mid\right.$ $\alpha \in \mathcal{T}\}$ is a function $E$ with domain $\cup\left\{D_{\alpha} \mid \alpha\right.$ is complex $\}$ such that $E(d) \subseteq$ $D_{\alpha_{1}} \times \cdots \times D_{\alpha_{n}}$ whenever $d \in D_{\left\langle\alpha_{1} \ldots \alpha_{n}\right\rangle}$.

The restriction of $E$ to $D_{\alpha}$ is written as $E_{\alpha}$, for any complex type $\alpha$, so that $E_{\alpha}: D_{\alpha} \rightarrow \mathcal{P}\left(D_{\alpha_{1}} \times \cdots \times D_{\alpha_{n}}\right)$ if $\alpha=\left\langle\alpha_{1} \ldots \alpha_{n}\right\rangle$.

The limiting case that $n=0$ is of some interest here. In this case the product $D_{\alpha_{1}} \times \cdots \times D_{\alpha_{n}}$ equals $\{\langle\rangle\}$. We identify \langle\rangle with $\varnothing, \varnothing$ with 0 , and $\{\varnothing\}$ with 1 , so that $E_{\langle\rangle}: D_{\langle\rangle} \rightarrow\{0,1\}$ if $E_{\langle\rangle}$is an extension function of type \langle\rangle for $D$. Note that, while the range of $E_{\langle\rangle}$thus consists of the two standard truth-values, the domain $D_{\langle\rangle}$of propositions can have any cardinality $\geq 2$. Propositions with the same truth-value need not be identified and, as will become apparent, even propositions that receive the same truth value in all structures need not be identical in any given structure. 
Definition 7. $A$ generalized frame for the language $\mathcal{L}$ is a triple $\langle D, I, E\rangle$ such that $D$ is a collection of domains, $I$ is an intension function for $D$ and $\mathcal{L}$, and $E$ is an extension function for $D$.

We are interested in the extensions $E\left(I\left(a, A_{\alpha}\right)\right)$ of terms $A$ of complex type $\alpha$. Let $V$ be the composition of $E$ and $I$, so that, in the interest of readability, we can write $V(a, A)$, for $E(I(a, A))$. The following definition, which gives the central notion of this paper, puts constraints on intension and extension functions that cause terms to get their usual semantic values.

Definition 8. A generalized frame $\langle D, I, E\rangle$ for $\mathcal{L}$ is an intensional model for $\mathcal{L}$ if

$$
\begin{aligned}
& \text { 1. } V(a, \perp)=0 \\
& \text { 2. } V(a, A B)=\{\langle\vec{d}\rangle \mid\langle I(a, B), \vec{d}\rangle \in V(a, A)\} \\
& \text { 3. } V\left(a, \lambda x_{\beta} \cdot A\right)=\left\{\langle d, \vec{d}\rangle \mid d \in D_{\beta} \text { and }\langle\vec{d}\rangle \in V(a[d / x], A)\right\} \\
& \text { 4. } V(a, A \subset B)=1 \Longleftrightarrow V(a, A) \subseteq V(a, B)
\end{aligned}
$$

To better understand the motivation behind the second and third clauses of this definition, it may help to consider that any $n+1$ place relation $R$ can be thought of as a unary function $F$ such that $F(d)=\{\langle\vec{d}\rangle \mid\langle d, \vec{d}\rangle \in R\}$. Thus $V(a, A B)=F(I(a, B))$, where $F$ is the function corresponding to $V(a, A)$ and $V\left(a, \lambda x_{\beta} . A\right)$ corresponds to the function $F$ such that $F(d)=V(a[d / x], A)$ for each $d \in D_{\beta}$. For further discussion of this little trick in an extensional setting see Muskens [18, 19.

If $M=\langle D, I, E\rangle$ is an intensional model, $a$ is an assignment for $D$, and $\varphi$ is a formula, we may alternatively write $M \models \varphi[a]$ for $V(a, \varphi)=1$. In case $\varphi$ is a sentence it makes sense to write $M \models \varphi$ if $M \models \varphi[a]$ for some $a$. The following facts are unsurprising but useful.

Proposition 1. Let $M=\langle D, I, E\rangle$ be an intensional model, and let $a$ be an assignment for $D$. Then, for all $\varphi, \psi, A, B$ and $B^{\prime}$ of appropriate types,

1. $V(a, \varphi \rightarrow \psi)=0$ iff $V(a, \varphi)=1$ and $V(a, \psi)=0$;

2. $V\left(a, \forall x_{\alpha} \varphi\right)=1$ iff $V(a[d / x], \varphi)=1$ for all $d \in D_{\alpha}$;

3. $V(a,(\lambda x . A) B)=V(a, A\{x:=B\})$, if $B$ is free for $x$ in $A$;

4. If $V(a, A=B)=1$ then $V(a, A \subset B)=1$;

5. $V(a, A=A)=1$;

6. If $V\left(a, B=B^{\prime}\right)=1$ then $V\left(a, A\{x:=B\}=A\left\{x:=B^{\prime}\right\}\right)=1$, provided $B$ and $B^{\prime}$ are free for $x$ in $A$.

Proof. Left to the reader. 
Note that $\beta$-conversion preserves extensional identity, but that it does not necessarily preserve intensional identity, i.e. $\left(\lambda x_{\alpha} \cdot A\right) B=A\{x:=B\}$ is not necessarily true given the usual side condition. Similar remarks can be made about $\eta$-conversion and even about $\alpha$-conversion. Since it is not necessary to hardwire these principles into the logic, we have chosen not to do so. However, the principles can clearly be added to the logic by means of an axiomatic extension. In section 7 below, where a linguistic application is considered, this axiomatic extension will be given.

The last two statements in proposition 1 above show that $=$ is the usual congruence, but intensional models may still have the undesirable property that $=$ does not denote true identity of intension. This is an anomaly we want to get rid of. Intensional models are called normal just in case they have the desired property.

Definition 9. An intensional model $M=\langle D, I, E\rangle$ is normal if, for any type $\alpha$, any $d, d^{\prime} \in D_{\alpha}$, and any $a,\left\langle d, d^{\prime}\right\rangle \in V\left(a, \lambda x_{\alpha} \lambda x_{\alpha}^{\prime} \cdot x=x^{\prime}\right)$ implies $d=d^{\prime}$.

That a restriction to normal intensional models does not buy us any new truths is shown by the next proposition. Its proof uses the Axiom of Choice unless $M$ is countable.

Proposition 2. Let $M$ be an intensional model. There is a normal intensional model $\bar{M}$ such that $M \models \varphi \Longleftrightarrow \bar{M} \models \varphi$ for each sentence $\varphi$.

Proof. Suppose $M=\langle D, I, E\rangle$. Let $\sim$ be the relation given by $d \sim d^{\prime}$ iff $\left\langle d, d^{\prime}\right\rangle \in$ $V\left(a, \lambda x_{\alpha} \lambda x_{\alpha}^{\prime} \cdot x=x^{\prime}\right)$ for any $d, d^{\prime} \in D_{\alpha}$ and any $\alpha$ (where $a$ is arbitrary). Clearly, $\sim$ is an equivalence relation. Note that, by proposition 1 and definition 5] for any term $A$,

$$
d \sim d^{\prime} \Longrightarrow I(a[d / x], A) \sim I\left(a\left[d^{\prime} / x\right], A\right) .
$$

Define $\bar{d}=\left\{d^{\prime} \mid d \sim d^{\prime}\right\}$, let $\bar{D}_{\alpha}=\left\{\bar{d} \mid d \in D_{\alpha}\right\}$, and let $\bar{D}=\left\{\bar{D}_{\alpha} \mid \alpha \in \mathcal{T}\right\}$. Let $f$ be a function such that $f(\bar{d}) \in \bar{d}$, if $\bar{d} \in \bar{D}_{\alpha}$. For any assignment $a$ for $\bar{D}$, let $a^{\circ}$ be the assignment for $D$ defined by $a^{\circ}(x)=f(a(x))$, for all $x$. Let $\bar{I}(a, A)=\overline{I\left(a^{\circ}, A\right)}$, for each assignment $a$ for $\bar{D}$ and each term $A$. Then $\bar{I}$ is an intension function for $\bar{D}$. The first three requirements of definition 5 are easily checked, so let us check the last requirement. Note that

$$
\begin{aligned}
I\left(a^{\circ}, A\{x:=B\}\right) & =\text { (Definition 5) } \\
I\left(a^{\circ}\left[I\left(a^{\circ}, B\right) / x\right], A\right) & \sim \text { (4) } \\
\left.I\left(a^{\circ}\left[f\left(\overline{I\left(a^{\circ}, B\right)}\right) / x\right]\right), A\right) & =\text { (definition of } \bar{I}) \\
\left.I\left(a^{\circ}[f(\bar{I}(a, B)) / x]\right), A\right) & =\text { (definition of } \circ) \\
I\left((a[\bar{I}(a, B) / x])^{\circ}, A\right) . &
\end{aligned}
$$

From this conclude that $\bar{I}(a, A\{x:=B\})=\bar{I}(a[\bar{I}(a, B) / x], A)$.

Define $\bar{E}$ by letting $\bar{E}\left(\bar{d}_{\alpha}\right)=\left\{\left\langle\overline{d_{1}}, \ldots, \overline{d_{n}}\right\rangle \mid\left\langle d_{1}, \ldots, d_{n}\right\rangle \in E(d)\right\}$, if $\alpha$ is complex. It is easy to see that this is well-defined. Since $\left\langle\overline{d_{1}}, \ldots, \overline{d_{n}}\right\rangle \in \bar{E}(\bar{I}(a, A))$ iff $\left\langle d_{1}, \ldots, d_{n}\right\rangle \in V\left(a^{\circ}, A\right)$ it follows that $\bar{M}=\langle\bar{D}, \bar{I}, \bar{E}\rangle$ is an intensional model, $M \models \varphi \Longleftrightarrow \bar{M} \models \varphi$ for each sentence $\varphi$, and $\bar{M}$ is normal. 


$$
\begin{aligned}
& \frac{\Pi \Rightarrow \Sigma}{\Pi^{\prime} \Rightarrow \Sigma^{\prime}}[W], \quad \text { if } \Pi \subseteq \Pi^{\prime}, \Sigma \subseteq \Sigma^{\prime} \\
& \overline{\Pi, \varphi \Rightarrow \Sigma, \varphi}[R] \quad \overline{\Pi, \perp \Rightarrow \Sigma}[\perp \mathrm{L}] \\
& \frac{\Pi, A\{x:=B\} \vec{C} \Rightarrow \Sigma}{\Pi,(\lambda x . A) B \vec{C} \Rightarrow \Sigma}[\lambda \mathrm{L}] \quad \frac{\Pi \Rightarrow \Sigma, A\{x:=B\} \vec{C}}{\Pi \Rightarrow \Sigma,(\lambda x . A) B \vec{C}}[\lambda \mathrm{R}] \\
& \text { if } B \text { is free for } x \text { in } A \quad \text { if } B \text { is free for } x \text { in } A \\
& \frac{\Pi, B \vec{C} \Rightarrow \Sigma \quad \Pi \Rightarrow \Sigma, A \vec{C}}{\Pi, A \subset B \Rightarrow \Sigma}[\subset \mathrm{L}] \quad \frac{\Pi, A \vec{c} \Rightarrow \Sigma, B \vec{c}}{\Pi \Rightarrow \Sigma, A \subset B}[\subset \mathrm{R}] \\
& \text { if the constants } \vec{c} \text { are fresh }
\end{aligned}
$$

Table 1: Gentzen rules for ITL.

Now that the situation with respect to normality and non-normality of intensional models has become clear, we can define our semantic notion of consequence.

Definition 10. An intensional model $M$ for $\mathcal{L}$ refutes a sequent $\Pi \Rightarrow \Sigma$ of $\mathcal{L}$ if $M \models \varphi$ for all $\varphi \in \Pi$ and $M \not \models \varphi$ for all $\varphi \in \Sigma$. A sequent $\Gamma$ is $\mathrm{i}$-valid if no intensional model for $\mathcal{L}$ refutes $\Gamma$. $\Pi$ i-entails $\Sigma, \Pi \models_{i} \Sigma$, if $\Pi \Rightarrow \Sigma$ is i-valid.

Let us take stock. We have defined a notion of intensional model following the two-stage pattern discussed in section 2. This is also the pattern followed in Fitting [10], but we have avoided the complex "abstraction designation functions" that are used there but do not seem to have a justification beyond the fact that they are needed in proofs. Intensional models are a further generalisation of Henkin models in the following sense. While in intensional models the functions

$$
E_{\left\langle\alpha_{1} \ldots \alpha_{n}\right\rangle}: D_{\left\langle\alpha_{1} \ldots \alpha_{n}\right\rangle} \rightarrow \mathcal{P}\left(D_{\alpha_{1}} \times \cdots \times D_{\alpha_{n}}\right)
$$

need neither be injective nor surjective, the usual Henkin models are essentially obtained if an injectivity requirement is imposed. An additional requirement of surjectivity brings us to a variant of the so-called standard models of type theory.

\section{Proof Theory}

We now provide the relation of i-entailment with what will turn out to be a syntactic equivalent. The rules in Table 1 for which the usual notational conventions apply, constitute a Gentzen sequent calculus for ITL. If $\Pi \Rightarrow \Sigma$ is 


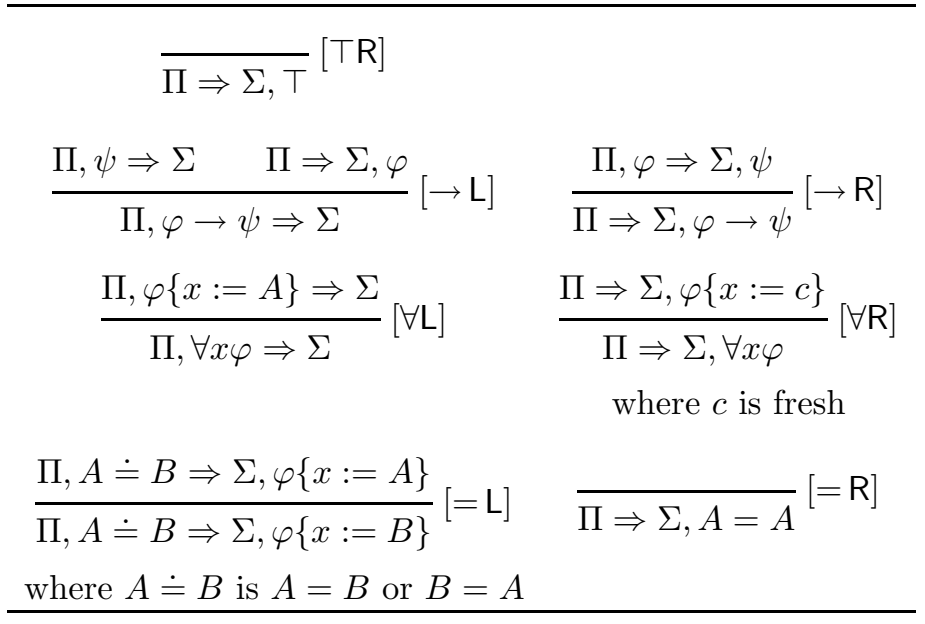

Table 2: Some classical rules derivable in ITL.

a (finite or infinite) sequent, then we say that $\Pi \Rightarrow \Sigma$ is provable, $\Pi \vdash \Sigma$, if there are finite $\Pi_{0} \subseteq \Pi$ and $\Sigma_{0} \subseteq \Sigma$ such that $\Pi_{0} \Rightarrow \Sigma_{0}$ can be proved in this calculus. The following theorem states that the calculus is sound.

Theorem 3 (Soundness). If a sequent $\Gamma$ is provable, $\Gamma$ is i-valid. Hence $\Pi \vdash \Sigma \Longrightarrow \Pi \models \models_{i} \Sigma$

Proof. Left to the reader. (The proof involves some observations about the behaviour of intension functions when the language is extended.)

That the converse (generalized completeness) also holds will be shown in the next section.

While the rules in Table 1 suffice to characterize the $\models_{i}$ relation, it is pleasant to also have the usual classical Gentzen rules for the defined connectives at one's disposal. These are available as derived rules. By way of example those for $T$, $\rightarrow, \forall$, and $=$ are given in Table 2 Given the abbreviations in definition 3 they are easily derivable from the ITL rules, as the reader may verify. Note that in view of the correctness of these rules it seems reasonable to say that ITL is indeed a classical logic.

\section{Model Existence}

The purpose of this section - which could be skipped on a first reading by readers mainly interested in the general characteristics of our logic - is to prove Generalized Completeness and some of its friends, such as the generalized LöwenheimSkolem and Compactness theorems. We will do this in the way Smullyan [27] did it for first-order logic, via a central Model Existence theorem from which the desired theorems all follow as corollaries. First it will be proved that certain 
"Hintikka" sequents, which can be thought of as resulting from a systematic but unsuccessful attempt to construct a Gentzen proof from the bottom up, are refutable. This is then used to show refutability of a wide class of sequents.

The definition of Hintikka sequents is close to that of the "Hintikka sets" in Smullyan [27] and Fitting [9, 10], but is also analogous to that of Schütte's [26] semi-valuations.

Definition 11. A sequent $\Gamma$ of $\mathcal{L}$ is called a Hintikka sequent in $\mathcal{L}$ if the following hold:

1. $\{\mathrm{L}: \varphi, \mathrm{R}: \varphi\} \nsubseteq \Gamma$ for any sentence $\varphi$;

2. $\mathrm{L}: \perp \notin \Gamma$;

3. $\mathrm{L}:(\lambda x . A) B \vec{C} \in \Gamma \Longrightarrow \mathrm{L}: A\{x:=B\} \vec{C} \in \Gamma$, if $\lambda x . A, B$, and the sequence of terms $\vec{C}$ are closed and of appropriate type;

4. R: $(\lambda x . A) B \vec{C} \in \Gamma \Longrightarrow \mathrm{R}: A\{x:=B\} \vec{C} \in \Gamma$, if $\lambda x . A, B$, and the sequence of terms $\vec{C}$ are closed and of appropriate type;

5. $\mathrm{L}: A \subset B \in \Gamma \Longrightarrow \mathrm{L}: B \vec{C} \in \Gamma$ or $\mathrm{R}: A \vec{C} \in \Gamma$, for all closed $A, B$ and sequences of closed $\vec{C}$ of appropriate types;

6. $\mathrm{R}: A \subset B \in \Gamma \Longrightarrow$ there are constants $\vec{c}$ of appropriate types such that $\{\mathrm{L}: A \vec{c}, \mathrm{R}: B \vec{c}\} \subseteq \Gamma$.

A Hintikka sequent $\Gamma$ in $\mathcal{L}$ is said to be complete if $\mathrm{L}: \varphi \in \Gamma$ or $\mathrm{R}: \varphi \in \Gamma$, for each sentence $\varphi$ of $\mathcal{L}$.

A key property of Hintikka sequents is that they are refuted by intensional models, as the following lemma shows. The intensional model constructed in its proof is closely akin to Andrews' $V$-complexes.

Lemma 4 (Hintikka Lemma). Let $\Gamma$ be a Hintikka sequent in a language $\mathcal{L}$ such that $\mathcal{L}_{\alpha} \neq \varnothing$ if $\alpha$ is basic. Then $\Gamma$ is refuted by an intensional model. If $\Gamma$ is complete, then $\Gamma$ is refuted by a normal countable intensional model.

Proof. Let $\Gamma$ be a Hintikka sequent in the language $\mathcal{L}$ as described. We will find an intensional model refuting $\Gamma$ using the Takahashi-Prawitz construction. The following induction on type complexity defines domains $D_{\alpha}$ as sets of pairs $\langle A, e\rangle$, where $A$ is a closed term of type $\alpha$ and $e$ is called a possible extension of A.

1. If $\alpha$ is basic let $D_{\alpha}=\left\{\langle c, c\rangle \mid c \in \mathcal{L}_{\alpha}\right\}$;

2. If $\alpha=\left\langle\alpha_{1} \ldots \alpha_{n}\right\rangle$ let $\left\langle A_{\alpha}, e\right\rangle \in D_{\alpha}$ iff $A$ is closed, $e \subseteq D_{\alpha_{1}} \times \cdots \times D_{\alpha_{n}}$ and, whenever $\left\langle B_{1}, e_{1}\right\rangle \in D_{\alpha_{1}}, \ldots,\left\langle B_{n}, e_{n}\right\rangle \in D_{\alpha_{n}}$

(a) If $\mathrm{L}: A B_{1} \ldots B_{n} \in \Gamma$ then $\left\langle\left\langle B_{1}, e_{1}\right\rangle, \ldots,\left\langle B_{n}, e_{n}\right\rangle\right\rangle \in e$;

(b) If R: $A B_{1} \ldots B_{n} \in \Gamma$ then $\left\langle\left\langle B_{1}, e_{1}\right\rangle, \ldots,\left\langle B_{n}, e_{n}\right\rangle\right\rangle \notin e$. 
It is worth observing that each $D_{\alpha}$ is a function if $\Gamma$ is complete. In that case each $D_{\alpha}$ will be countable.

The set $D=\left\{D_{\alpha} \mid \alpha \in \mathcal{T}\right\}$ will be the collection of domains of the refuting intensional model we are after. Note that, since each term has a unique type, the $D_{\alpha}$ are pairwise disjoint. The $D_{\alpha}$ are also non-empty. This follows from the assumption that $\mathcal{L}_{\alpha} \neq \varnothing$ in case $\alpha$ is basic; in case $\alpha=\left\langle\alpha_{1} \ldots \alpha_{n}\right\rangle$ it is easy to show that $\left\langle\lambda x_{\alpha_{1}} \ldots \lambda x_{\alpha_{n}} . \perp, \varnothing\right\rangle \in D_{\alpha}$.

We will define a function $I$ which will turn out to be an intension function for $D$. First some handy notation. If $\pi$ is an ordered pair, write $\pi^{1}$ and $\pi^{2}$ for the first and second elements of $\pi$ respectively, so that $\pi=\left\langle\pi^{1}, \pi^{2}\right\rangle$. If $f$ is a function whose values are ordered pairs, write $f^{1}$ and $f^{2}$ for the functions with the same domain as $f$, such that $f^{1}(z)=(f(z))^{1}$ and $f^{2}(z)=(f(z))^{2}$ for any argument $z$. Let $a$ be an assignment for $D$. The substitution $\overleftarrow{a}$ is defined by $\overleftarrow{a}(x)=a^{1}(x)$ and we let $I^{1}(a, A)=A \overleftarrow{a}$ for any term $A$. The second component of $I$, is defined by letting $I^{2}=\bigcup_{\alpha \in \mathcal{T}} I_{\alpha}^{2}$, where the $I_{\alpha}^{2}$ are functions such that $I_{\alpha}^{2}: \mathcal{A}_{D} \times T_{\alpha}^{\mathcal{L}} \rightarrow T_{\alpha}^{\mathcal{L}}$ if $\alpha \in \mathcal{B}$ and

$$
I_{\alpha}^{2}: \mathcal{A}_{D} \times T_{\alpha}^{\mathcal{L}} \rightarrow \mathcal{P}\left(D_{\alpha_{1}} \times \cdots \times D_{\alpha_{n}}\right)
$$

if $\alpha=\left\langle\alpha_{1} \ldots \alpha_{n}\right\rangle$. The $I_{\alpha}^{2}$ in their turn are defined using the following induction on the complexity of terms.

1. $I_{\alpha}^{2}\left(a, x_{\alpha}\right)=a^{2}(x)$, if $x$ is a variable; $I_{\alpha}^{2}\left(a, c_{\alpha}\right)=c$, if $\alpha$ is basic; $I_{\alpha}^{2}\left(a, c_{\alpha}\right)=\left\{\left\langle\left\langle A_{1}, e_{1}\right\rangle, \ldots,\left\langle A_{n}, e_{n}\right\rangle\right\rangle \mid\left\langle A_{i}, e_{i}\right\rangle \in D_{\alpha_{i}} \& \mathrm{~L}: c A_{1} \ldots A_{n} \in \Gamma\right\}$, if $\alpha=\left\langle\alpha_{1} \ldots \alpha_{n}\right\rangle$;

2. $I_{\langle\rangle}^{2}(a, \perp)=0$

3. $I_{\langle\vec{\alpha}\rangle}^{2}\left(a, A_{\langle\beta \vec{\alpha}\rangle} B_{\beta}\right)=\left\{\langle\vec{d}|\left\langle\left\langle I^{1}(a, B), I_{\beta}^{2}(a, B)\right\rangle, \vec{d}\right\rangle \in I_{\langle\beta \vec{\alpha}\rangle}^{2}(a, A)\right\}$

4. $I_{\langle\beta \vec{\alpha}\rangle}^{2}\left(a, \lambda x_{\beta} A_{\langle\vec{\alpha}\rangle}\right)=\left\{\langle d, \vec{d}\rangle \mid d \in D_{\beta} \&\langle\vec{d}\rangle \in I_{\langle\vec{\alpha}\rangle}^{2}(a[d / x], A)\right\}$

5. $I_{\langle\rangle}^{2}(a, A \subset B)=1 \Longleftrightarrow I_{\alpha}^{2}\left(a, A_{\alpha}\right) \subseteq I_{\alpha}^{2}\left(a, B_{\alpha}\right)$

Note that this definition does not depend on the question whether $I$ is an intension function for $D$ and $\mathcal{L}$, and indeed the latter is not immediately obvious. We need to check the requirements in definition [5] That $I(a, x)=a(x)$ for any variable $x$ is immediate and that $I(a, A)=I\left(a^{\prime}, A\right)$ if $a$ and $a^{\prime}$ agree on the variables free in $A$ follows by a standard property of substitutions and an easy induction. Suppose that $B$ is free for $x$ in $A$. Then

$$
\begin{aligned}
& I^{1}(a, A\{x:=B\})=A\{x:=B\} \overleftarrow{a}=A \overleftarrow{a}[x:=B \overleftarrow{a}]= \\
& A \overleftarrow{a}\left[x:=I^{1}(a, B)\right]=A \overleftarrow{a[I(a, B) / x]}=I^{1}(a[I(a, B) / x], A)
\end{aligned}
$$

That $I^{2}(a, A\{x:=B\})=I^{2}(a[I(a, B) / x], A)$ if $B$ is free for $x$ in $A$ follows by a straightforward induction on the complexity of $A$ which we leave to the reader. Thus $I(a, A\{x:=B\})=I(a[I(a, B) / x], A)$ if $B$ is free for $x$ in $A$. 
It remains to be shown that $I(a, A) \in D_{\alpha}$ for any assignment $a$ and term $A$ of type $\alpha$. This is done by induction on the complexity of $A$. That $I\left(a, x_{\alpha}\right) \in D_{\alpha}$ if $x$ is a variable follows from the fact that $I(a, x)=a(x)$ and that $I\left(a, c_{\alpha}\right) \in D_{\alpha}$ if $\alpha$ is basic is immediate. In the remaining cases the type of $A$ is complex and it suffices to prove that whenever $\alpha=\left\langle\alpha_{1} \ldots \alpha_{n}\right\rangle,\left\langle B_{1}, e_{1}\right\rangle \in D_{\alpha_{1}}, \ldots$, and $\left\langle B_{n}, e_{n}\right\rangle \in D_{\alpha_{n}}$ :

(a) If L: $A \overleftarrow{a} B_{1} \ldots B_{n} \in \Gamma$ then $\left\langle\left\langle B_{1}, e_{1}\right\rangle, \ldots,\left\langle B_{n}, e_{n}\right\rangle\right\rangle \in I^{2}(a, A)$

(b) If R: $A \overleftarrow{a} B_{1} \ldots B_{n} \in \Gamma$ then $\left\langle\left\langle B_{1}, e_{1}\right\rangle, \ldots,\left\langle B_{n}, e_{n}\right\rangle\right\rangle \notin I^{2}(a, A)$.

We shall consider each case. IH will be short for 'induction hypothesis'.

- $A_{\alpha} \equiv c$ and $\alpha=\left\langle\alpha_{1} \ldots \alpha_{n}\right\rangle$. The requirement follows from the definition of $I^{2}(a, c)$ and clause 1 . of definition 11

- $A_{\alpha} \equiv \perp$ and $\alpha=\langle\rangle$. The (a) part of the property follows from clause 2 . of definition 11 the (b) part from the fact that $I^{2}(a, \perp)=0=\varnothing$.

- $A \equiv B_{\left\langle\beta \alpha_{1} \ldots \alpha_{n}\right\rangle} C_{\beta}$. Suppose $\left\langle B_{1}, e_{1}\right\rangle \in D_{\alpha_{1}}, \ldots,\left\langle B_{n}, e_{n}\right\rangle \in D_{\alpha_{n}}$, then

$$
\begin{aligned}
\mathrm{L}:(B C) \overleftarrow{a} B_{1} \ldots B_{n} \in \Gamma & \Longleftrightarrow \\
\mathrm{L}: B \overleftarrow{a} C \overleftarrow{a} B_{1} \ldots B_{n} \in \Gamma \quad \Longrightarrow & (\mathrm{IH}) \\
\left\langle I(a, C),\left\langle B_{1}, e_{1}\right\rangle, \ldots,\left\langle B_{n}, e_{n}\right\rangle\right\rangle \in I^{2}(a, B) & \Longleftrightarrow \quad \text { (def. of } I) \\
\left\langle\left\langle B_{1}, e_{1}\right\rangle, \ldots,\left\langle B_{n}, e_{n}\right\rangle\right\rangle \in I^{2}(a, B C) &
\end{aligned}
$$

This proves the (a) part of the case; the (b) part is similar.

- $A \equiv\left(\lambda x_{\alpha_{1}} C_{\left\langle\alpha_{2} \ldots \alpha_{n}\right\rangle}\right)$. Again suppose $d_{1}=\left\langle B_{1}, e_{1}\right\rangle \in D_{\alpha_{1}}, \ldots, d_{n}=$ $\left\langle B_{n}, e_{n}\right\rangle \in D_{\alpha_{n}}$, and reason as follows.

$$
\begin{aligned}
\mathrm{R}:(\lambda x . C) \overleftarrow{a} B_{1} \ldots B_{n} \in \Gamma & \Longleftrightarrow \\
\mathrm{R}: \lambda x \cdot C \overleftarrow{a}_{x} B_{1} \ldots B_{n} \in \Gamma & \Longrightarrow \text { Def. [1] } B_{1} \text { is closed } \\
\mathrm{R}: C \overleftarrow{a}_{x}\left\{x:=B_{1}\right\} B_{2} \ldots B_{n} \in \Gamma & \Longleftrightarrow \\
\mathrm{R}: C \overleftarrow{a\left[d_{1} / x\right]} B_{2} \ldots B_{n} \in \Gamma & \Longrightarrow \text { (IH) } \\
\left\langle d_{2}, \ldots, d_{n}\right\rangle \notin I^{2}\left(a\left[d_{1} / x\right], C\right) & \Longleftrightarrow \text { (def. of } I) \\
\left\langle d_{1}, d_{2}, \ldots, d_{n}\right\rangle \notin I^{2}(a, \lambda x . C) &
\end{aligned}
$$

This proves the (b) part, which is similar to the (a) part.

- $A_{\alpha} \equiv B \subset C$. Then $\alpha=\langle\rangle$ and $B$ and $C$ have some type $\left\langle\alpha_{1} \ldots \alpha_{n}\right\rangle$. Using induction we may assume that $I(a, B), I(a, C) \in D_{\left\langle\alpha_{1} \ldots \alpha_{n}\right\rangle}$. Suppose $\mathrm{L}:(B \subset C) \overleftarrow{a} \in \Gamma$, i.e. L: $B \overleftarrow{a} \subset C \overleftarrow{a} \in \Gamma$ and reason as follows.

$$
\begin{array}{rll}
\left\langle\left\langle B_{1}, e_{1}\right\rangle, \ldots,\left\langle B_{n}, e_{n}\right\rangle\right\rangle \in I^{2}(a, B) & \Longrightarrow & (\mathrm{IH}) \\
\mathrm{R}: B \overleftarrow{a} B_{1} \ldots B_{n} \notin \Gamma & \Longrightarrow & \text { (Def. 111) } \\
\mathrm{L}: C \overleftarrow{a} B_{1} \ldots B_{n} \in \Gamma & \Longrightarrow & \text { (IH) } \\
\left\langle\left\langle B_{1}, e_{1}\right\rangle, \ldots,\left\langle B_{n}, e_{n}\right\rangle\right\rangle \in I^{2}(a, C) & &
\end{array}
$$


We conclude that $I^{2}(a, B) \subseteq I^{2}(a, C)$ and that $I^{2}(a, B \subset C)=1$. This proves the (a) part of the property. The (b) part is left to the reader.

This concludes the proof that $I$ is an intension function for $D$ and $\mathcal{L}$. Now define the function $E$ by letting $E(\langle A, e\rangle)=e$ if $\langle A, e\rangle \in D_{\alpha}$ for any complex $\alpha$. Clearly, $E(I(a, A))=I^{2}(a, A)$ for any $A_{\alpha}, E$ is an extension function for $D$, and $M=\langle D, I, E\rangle$ is an intensional model for the language $\mathcal{L}$. It is easy to see that $M$ refutes $\Gamma$. We have already established that $M$ is countable if $\Gamma$ is complete, and proposition 2 gives a normal intensional model refuting $\Gamma$ which is countable in case $\Gamma$ is complete.

Before we continue with the proof of Model Existence, let us look at an application. Hintikka's Lemma sometimes gives an easy way of showing the refutability of certain sequents. For example, while standard higher order logic validates the sentence $p=q \vee q=r \vee r=p$ (where $p, q$ and $r$ are type \langle\rangle constants), a Hintikka sequent (corresponding to an open tableau branch) shows that this is not the case in ITL.

Proposition 5. $\Rightarrow p=q, q=r, r=p$ is refutable by an intensional model.

Proof. The non-abbreviated form of $\Rightarrow p=q, q=r, r=p$ is

$\Gamma=\{\mathrm{R}:(\lambda z . \top) \subset(\lambda z . z p \subset z q), \mathrm{R}:(\lambda z . \top) \subset(\lambda z . z q \subset z r)$,

$$
\mathrm{R}:(\lambda z . \top) \subset(\lambda z . z r \subset z p)\},
$$

with $z$ a variable of type $\langle\langle\rangle\rangle$. An inspection tells that the following extension $\Gamma^{+}$of $\Gamma$, in which $c_{1}, c_{2}$ and $c_{3}$ are constants of type $\langle\langle\rangle\rangle$, is a Hintikka sequent.

$\left\{\mathrm{R}:(\lambda z . \top) \subset(\lambda z . z p \subset z q), \mathrm{L}:(\lambda z . \top) c_{1}, \mathrm{R}:(\lambda z . z p \subset z q) c_{1}, \mathrm{~L}: \top\right.$,

$\mathrm{R}: c_{1} p \subset c_{1} q, \mathrm{~L}: c_{1} p, \mathrm{R}: c_{1} q, \mathrm{R}:(\lambda z \cdot \top) \subset(\lambda z . z q \subset z r), \mathrm{L}:(\lambda z \cdot \top) c_{2}$,

$\mathrm{R}:(\lambda z . z q \subset z r) c_{2}, \mathrm{R}: c_{2} q \subset c_{2} r, \mathrm{~L}: c_{2} q, \mathrm{R}: c_{2} r$,

$\mathrm{R}:(\lambda z . \top) \subset(\lambda z . z r \subset z p), \mathrm{L}:(\lambda z . \top) c_{3}, \mathrm{R}:(\lambda z . z r \subset z p) c_{3}, \mathrm{R}: c_{3} r \subset c_{3} p$,

$\left.\mathrm{L}: c_{3} r, \mathrm{R}: c_{3} p\right\}$

It follows that $\Gamma^{+}$and hence $\Gamma$ are refutable by an intensional model.

This shows that it is consistent to assume that there are at least three propositions. It is clear that the method can be generalized to show that it is consistent to assume a set of propositions $\geq$ any given countable cardinality. If the countability restriction on languages is dropped, the existence of intensional models with type \langle\rangle domains $\geq$ any given cardinality is obtained. We leave it to the reader to show that $p \leftrightarrow q \Rightarrow p=q$ and other instances of Extensionality are refutable.

Let us return to the main line of argument. In order to state the model existence theorem below, we need the notion of a provability property (closely related to Smullyan's [27] abstract consistency property).

Definition 12. Let $\mathcal{P}$ be a set of sequents in the language $\mathcal{L} . \mathcal{P}$ is a provability property in $\mathcal{L}$ if $\mathcal{P}$ is closed under sequent rules, i.e. if $\Gamma \in \mathcal{P}$ whenever $\left\{\Gamma_{1}, \ldots, \Gamma_{n}\right\} \subseteq \mathcal{P}$ and $\Gamma_{1}, \ldots, \Gamma_{n} / \Gamma$ is a sequent rule. 
A provability property $\mathcal{P}$ in $\mathcal{L}$ is sound if no $\Gamma \in \mathcal{P}$ is refuted by an intensional model for $\mathcal{L}$.

We now come to Model Existence itself: sequents that are not elements of a sound provability property (in an extended language) can be extended to Hintikka sequents (in that language) and are hence refutable.

Theorem 6 (Model Existence). Let $\mathcal{L}$ and $\mathcal{C}$ be languages such that $\mathcal{L} \cap \mathcal{C}=\varnothing$ and each $\mathcal{C}_{\alpha}$ is denumerably infinite. Assume that $\mathcal{P}$ is a sound provability property in $\mathcal{L} \cup \mathcal{C}$ and that $\Gamma$ is a sequent in the language $\mathcal{L}$. If $\Gamma \notin \mathcal{P}$ then $\Gamma$ is refuted by a countable normal intensional model.

Proof. Let $\mathcal{P}$ and $\Gamma$ be as described. We construct a Hintikka sequent $\Gamma^{*}$ such that $\Gamma \subseteq \Gamma^{*}$. Let $\vartheta_{1}, \ldots, \vartheta_{n}, \ldots$ be an enumeration of all signed sentences in $\mathcal{L} \cup \mathcal{C}$. Write $\iota(\vartheta)$ for the index that the signed sentence $\vartheta$ obtains in this enumeration. Let $\Gamma_{0}=\Gamma$ and define each $\Gamma_{n+1}$ by distinguishing the following cases.

- $\Gamma_{n+1}=\Gamma_{n}$, if $\Gamma_{n} \cup\left\{\vartheta_{n}\right\} \in \mathcal{P}$;

- $\Gamma_{n+1}=\Gamma_{n} \cup\left\{\vartheta_{n}\right\}$, if $\Gamma_{n} \cup\left\{\vartheta_{n}\right\} \notin \mathcal{P}$ and $\vartheta_{n}$ is not of the form R: $A \subset B$;

- $\Gamma_{n+1}=\Gamma_{n} \cup\left\{\vartheta_{n}, \mathrm{~L}: A c_{1} \ldots c_{n}, \mathrm{R}: B c_{1} \ldots c_{n}\right\}$, if $\Gamma_{n} \cup\left\{\vartheta_{n}\right\} \notin \mathcal{P}$ and $\vartheta_{n}=$ R: $A \subset B$ for $A$ and $B$ of type $\left\langle\alpha_{1} \ldots \alpha_{n}\right\rangle$, where each $c_{i}$ is the first constant in $\mathcal{C}_{\alpha_{i}}$ which does not occur in $\Gamma_{n} \cup\left\{\vartheta_{n}\right\}$ and is no element of $\left\{c_{1}, \ldots, c_{i-1}\right\}$

This is well-defined since each $\Gamma_{n}$ contains only a finite number of constants from $\mathcal{C}$. That $\Gamma_{n} \notin \mathcal{P}$ for each $n$ follows by a simple induction which uses the definition of a provability property and the fact that $[\subset R]$ is a sequent rule. Define $\Gamma^{*}=\bigcup_{n} \Gamma_{n}$. For all finite sets $\left\{\vartheta_{k_{1}}, \ldots, \vartheta_{k_{n}}\right\}$ and for all $k \geq$ $\max \left\{k_{1}, \ldots, k_{n}\right\}$

$$
\left\{\vartheta_{k_{1}}, \ldots, \vartheta_{k_{n}}\right\} \subseteq \Gamma^{*} \Leftrightarrow \Gamma_{k} \cup\left\{\vartheta_{k_{1}}, \ldots, \vartheta_{k_{n}}\right\} \notin \mathcal{P}
$$

In order to show this, let $k \geq \max \left\{k_{1}, \ldots, k_{n}\right\}$ and let $\left\{\vartheta_{k_{1}}, \ldots, \vartheta_{k_{n}}\right\} \subseteq \Gamma^{*}$. Then there is some $\ell$ such that $\left\{\vartheta_{k_{1}}, \ldots, \vartheta_{k_{n}}\right\} \subseteq \Gamma_{\ell}$. Let $m=\max \{k, \ell\}$. We have that $\Gamma_{k} \cup\left\{\vartheta_{k_{1}}, \ldots, \vartheta_{k_{n}}\right\} \subseteq \Gamma_{m}$. Since $\Gamma_{m} \notin \mathcal{P}$ and $\mathcal{P}$ is closed under supersets (rule $[W]$ ), it follows that $\Gamma_{k} \cup\left\{\vartheta_{k_{1}}, \ldots, \vartheta_{k_{n}}\right\} \notin \mathcal{P}$. For the reverse direction, suppose that $\Gamma_{k} \cup\left\{\vartheta_{k_{1}}, \ldots, \vartheta_{k_{n}}\right\} \notin \mathcal{P}$. Then, since $\mathcal{P}$ is closed under supersets, $\Gamma_{k_{i}} \cup\left\{\vartheta_{k_{i}}\right\} \notin \mathcal{P}$, for each of the $k_{i}$. By the construction of $\Gamma^{*}$ each $\vartheta_{k_{i}} \in \Gamma^{*}$ and $\left\{\vartheta_{k_{1}}, \ldots, \vartheta_{k_{n}}\right\} \subseteq \Gamma^{*}$.

With the help of (5) it can be verified that $\Gamma^{*}$ is a Hintikka sequent. The last condition of Definition [11 immediately follows from the construction of $\Gamma^{*}$. We check condition 5, which may serve as an example for the other cases. Assume $\mathrm{L}: A \subset B \in \Gamma^{*}$ and let $k$ be the maximum of $\iota(\mathrm{L}: A \subset B), \iota(\mathrm{L}: B \vec{C})$, and $\iota(\mathrm{R}: A \vec{C})$. Since, by (5), $\Gamma_{k} \cup\{\mathrm{L}: A \subset B\} \notin \mathcal{P}$ and since $\mathcal{P}$ is closed under sequent rules, it must be the case that either $\Gamma_{k} \cup\{\mathrm{L}: B \vec{C}\} \notin \mathcal{P}$ or $\Gamma_{k} \cup\{\mathrm{R}: A \vec{C}\} \notin \mathcal{P}$, Using (5), we find that $\mathrm{L}: B \vec{C} \in \Gamma^{*}$ or $\mathrm{R}: A \vec{C} \in \Gamma^{*}$. 
We conclude that $\Gamma^{*}$ is refuted by an intensional model $M$. In order to prove that there is a normal countable intensional model that refutes $\Gamma^{*}$ and hence $\Gamma$ it suffices to show that $\Gamma^{*}$ is complete. Let $\varphi$ be any sentence of $\mathcal{L} \cup \mathcal{C}$ and assume that $\mathrm{L}: \varphi \notin \Gamma^{*}$ and $\mathrm{R}: \varphi \notin \Gamma^{*}$. Then, by (5), $\Gamma_{k} \cup\{\mathrm{L}: \varphi\} \in \mathcal{P}$ and $\Gamma_{k} \cup\{\mathrm{R}: \varphi\} \in \mathcal{P}$, for sufficiently large $k$. But $M$ refutes $\Gamma_{k}$ and therefore must either refute $\Gamma_{k} \cup\{\mathrm{L}: \varphi\}$ or $\Gamma_{k} \cup\{\mathrm{R}: \varphi\}$, contradicting the soundness of $\mathcal{P}$. Thus $\Gamma^{*}$ is complete and some normal countable intensional model refutes $\Gamma^{*}$ and $\Gamma$.

From model existence we can derive some nice corollaries. In the following $\Gamma$ will always be a sequent in some language $\mathcal{L}$ while $\Delta$ ranges over sequents in $\mathcal{L} \cup \mathcal{C}$, where $\mathcal{L}$ and $\mathcal{C}$ are as in the formulation of Theorem 6

Corollary 7 (Generalized Compactness). If $\Gamma$ is $i$-valid then some finite $\Gamma_{0} \subseteq \Gamma$ is i-valid.

Proof. $\left\{\Delta \mid\right.$ some finite $\Delta_{0} \subseteq \Delta$ is i-valid $\}$ is a sound provability property.

Corollary 8 (Generalized Löwenheim-Skolem). If $\Gamma$ is not i-valid then $\Gamma$ is refutable by a countable normal intensional model.

Proof. $\{\Delta \mid \Delta$ is i-valid $\}$ is a sound provability property.

Corollary 9 (Generalized Completeness). If $\Gamma$ is i-valid then $\Gamma$ is provable. Hence $\Pi \models_{i} \Sigma \Longrightarrow \Pi \vdash \Sigma$.

Proof. $\{\Delta \mid \Delta$ is provable $\}$ is a sound provability property.

Corollary 10 (Cut elimination). If $\Pi, \varphi \vdash \Sigma$ and $\Pi \vdash \Sigma, \varphi$ then $\Pi \vdash \Sigma$.

Proof. Use soundness and completeness.

\section{A Linguistic Application}

We now turn to a linguistic application of ITL and will develop the semantics of a tiny fragment of English containing verbs of propositional attitude. It will be shown that, given the present logic, it is consistent for an agent $a$ to know that $\varphi$ without knowing that $\psi$, even if $\varphi$ and $\psi$ are co-entailing.

Before considering our special application, however, let us address the general point of axiomatic extensions of the base logic. In most applications one will like to work with a subclass of the class of intensional models that conform to some set of non-logical axioms $\mathcal{S}$. In that case one can define $\Pi \models_{\mathcal{S}} \Sigma$ to be $\mathcal{S} \cup \Pi \models_{i} \Sigma$, while $\Pi \vdash_{\mathcal{S}} \Sigma$ can be defined as $\mathcal{S} \cup \Pi \vdash \Sigma$. Soundness and generalized completeness immediately give that $\Pi \models_{\mathcal{S}} \Sigma \Longleftrightarrow \Pi \vdash_{\mathcal{S}} \Sigma$. Not all applications will instantiate $\mathcal{S}$ in the same way, but one set of axioms that immediately come to mind, and that we shall adopt here, are the usual principles of $\lambda$-conversion. We may add these by assuming that $\mathcal{S}$ contains all universal closures of instantiations of the following schemes. 


\begin{tabular}{|c|c|c|c|}
\hline WORD & TRANSLATION & WORD & TRANSLATION \\
\hline if & $\lambda p_{\langle\rangle} \lambda q_{\langle\rangle} \cdot p \rightarrow q$ & $\operatorname{man}$ & $\operatorname{man}_{\langle e\rangle}$ \\
\hline no & $\lambda P_{\langle e\rangle}^{\prime} \lambda P_{\langle e\rangle} \cdot \neg \exists x_{e}\left(P^{\prime} x \wedge P x\right)$ & unicorn & unicorn $_{\langle e\rangle}$ \\
\hline some & $\lambda P_{\langle e\rangle}^{\prime} \lambda P_{\langle e\rangle} \cdot \exists x_{e}\left(P^{\prime} x \wedge P x\right)$ & runs & $\operatorname{run}_{\langle e\rangle}$ \\
\hline every & $\lambda P_{\langle e\rangle}^{\prime} \lambda P_{\langle e\rangle} \cdot \forall x_{e}\left(P^{\prime} x \rightarrow P x\right)$ & laughs & $\operatorname{laugh}_{\langle e\rangle}$ \\
\hline loves & $\lambda Q_{\langle\langle e\rangle\rangle} \lambda x_{e} \cdot Q\left(\lambda y_{e}\right.$. love $\left._{\langle e e\rangle} x y\right)$ & Bill & $\operatorname{bill}_{\langle\langle e\rangle\rangle}$ \\
\hline is & $\lambda Q_{\langle\langle e\rangle\rangle} \lambda x_{e} \cdot Q\left(\lambda y_{e} \cdot x=y\right)$ & Ann & $a n n_{\langle\langle e\rangle\rangle}$ \\
\hline knows & $\lambda p_{\langle\rangle} \lambda x_{e} \cdot k n o w_{\langle e\langle\rangle\rangle} x p$ & Tully & tully $\langle\langle e\rangle\rangle$ \\
\hline believes & $\lambda p_{\langle\rangle} \lambda x_{e}$. believe $_{\langle e\langle\rangle\rangle} x p$ & Cicero & cicero $\langle\langle e\rangle\rangle$ \\
\hline
\end{tabular}

Table 3: Some words and their translations

( $\alpha) \lambda x \cdot A=\lambda y \cdot A\{x:=y\}$, if $y$ is free for $x$ in $A$;

( $\beta)(\lambda x . A) B=A\{x:=B\}$, if $B$ is free for $x$ in $A$;

$(\eta) \lambda x \cdot A x=A$, if $x$ is not free in $A$.

As soon as these schemes are added to the base logic, the result is full intensional identity of $\beta \eta$ equivalent terms, i.e. $\models_{\mathcal{S}} A=B$ will hold if $A={ }_{\beta \eta} B$.

For our linguistic application we will proceed along lines pioneered by Montague [16] and define a small fragment of English. The words of this fragment are given in Table 3 along with their translations into type logic. In these translations the terms love, run, man, etc. are constants of the types indicated, where $e$ is the type of entities. The set of syntactic structures is obtained by stipulating that all words in Table 3 are syntactic structures and that $[X Y]$ is a syntactic structure whenever $X$ and $Y$ are syntactic structures. Defining syntactic structures in this way leads to a lot of gibberish along with the structures we are interested in, but this is not important for present purposes. As long as the desired structures are there and get reasonable interpretations our aim is served.

Let us define the relation $\rightsquigarrow$ ("translates as") between syntactic structures and terms as the smallest relation such that 1) $X \rightsquigarrow A$ if $X$ is a word and $A$ is its translation in Table 3 and 2) if $X \rightsquigarrow A$ and $Y \rightsquigarrow B$ then [XY] is a well-formed term and $[X Y] \rightsquigarrow B A$ if $B A$ is well-formed. This leaves open the possibility that a syntactic structure does not get a translation and indeed many do not. Structures $X$ for which there is no $A$ such that $X \rightsquigarrow A$ are called uninterpretable and we have no interest in them.

Let us turn to some syntactic structures that are interpretable. In (10) below two are given, together with (the $\beta$ normal forms of) their interpretations. Clearly, (10), the interpretation of (10), i-entails and is i-entailed by (10), which is the interpretation of (11).

(1) a. [[[no man]laughs][if[[some unicorn]runs]]]

b. $\exists x($ unicorn $x \wedge \operatorname{run} x) \rightarrow \neg \exists x(\operatorname{man} x \wedge \operatorname{laugh} x)$ 
c. [[[no unicorn]runs] [if[[some man]laughs]]]

d. $\exists x(\operatorname{man} x \wedge$ laugh $x) \rightarrow \neg \exists x($ unicorn $x \wedge$ run $x)$

This does not mean however that (10) and (1-d) are identical in all intensional models, as nothing excludes the possibility that $I(a, \mathbb{1} b)) \neq I(a, \mathbb{1} l))$ for some intension function $I$. It follows that the two structures in (2) are not co-entailing.

(2) a. [[every man] $[$ knows [[[no man]laughs] [if[[some unicorn]runs]]]]]

b. $\forall y(\operatorname{man} y \rightarrow$ know $y(\exists x($ unicorn $x \wedge \operatorname{run} x) \rightarrow \neg \exists x(\operatorname{man} x \wedge$ laugh $x)))$

c. [[every man] [knows[[[no unicorn]runs][if[[some man]laughs]]]]]

d. $\forall y(\operatorname{man} y \rightarrow$ know $y(\exists x(\operatorname{man} x \wedge \operatorname{laugh} x) \rightarrow \neg \exists x($ unicorn $x \wedge$ run $x)))$

Suppose that $c$ is some constant of type $e$. Then know $c(\mathbb{1}) \Rightarrow k n o w c$ (1) is in fact a Hintikka sequent and is therefore refuted by an intensional model (addition of $(\alpha),(\beta)$ and $(\eta)$ does not change this). This intensional model can also be used to show that (2b) does not entail (2a). This is as desired, for even if (27) holds there may well be a man who has not managed to draw the inference necessary to arrive at (15). We have thus shown that the logic avoids the problem of logical omniscience in the sense that it does not exclude the possibility that a person knows one thing but fails to know another thing logically equivalent with it. Essential use was made of the failure of Extensionality in our logic ITL: terms of complex type can have the same extensions, even in all intensional models, without necessarily having the same intension.

This distinction between extension and intension does not extend to terms of basic type however and this raises the question how names are to be dealt with. If they are treated straightforwardly using constants of type $e$ (e.g. $b_{e}$, or in the present context preferably $\lambda P . P b$, for 'Bill') we run into the standard problems of the 'Cicero-Tully' or 'Hesperus-Phosphorus' kind. However, there are many reasonable translations that do not directly equate names with type $e$ constants. The translations in Table 3 that send names to constants of the quantifier type $\langle\langle e\rangle\rangle$, may serve as an example, provided some meaning postulates (additions to $\mathcal{S}$ ) like the following are adopted.

(3) a. $\forall P(\operatorname{ann} P \leftrightarrow P a)$

b. $\forall P(b i l l P \leftrightarrow P b)$

c. $\forall P($ tully $P \leftrightarrow P t)$

d. $\forall P($ cicero $P \leftrightarrow P c)$

The structure [Tully runs] translates as tully run, but given the meaning postulates just introduced, this is equivalent with run $t$. Similarly, [Cicero runs] translates as cicero run, equivalent with run $c$. And since [Tully[is Cicero]] is translated as $\operatorname{tully}(\lambda x$.cicero $(\lambda y \cdot x=y))$, which is equivalent with $t=c$, it 
is readily explained why the argument Tully runs, Tully is Cicero, therefore Cicero runs holds. But this reasoning essentially depends on extensional equivalence and therefore will not go through once propositional attitudes enter the picture. Consider the structure [Ann[believes[Tully runs]]]. It translates as ann $(\lambda x$.believe $x($ tully run $)$ ) and this is equivalent with believe a (tully run), while believe a (cicero run) is equivalent with the translation of [Ann[believes [Cicero runs]]]. However, there is no co-entailment between these sentences, even in the presence of the postulates in (3) and the translation of [Tully[is Cicero]].

This shows that even for names the sense/reference distinction can be captured in this logic, provided one is willing to treat names with the help of predicates (Quine's 'primacy of predicates' comes to mind). Treating them as being of type $\langle\langle e\rangle\rangle$, as we have done here, is one possible strategy. There may be others.

The present application of our intensional type theory to linguistic semantics has avoided the concept of possible worlds altogether, as it was not needed in order to illustrate our points. However, as possible worlds are obviously extremely useful for the analysis of a range of natural language constructions (though not for the true intensionality we have been concerned with in this paper), one might well want to combine them with the present approach. Muskens [19, chapter 4] gives a translation of what is essentially the fragment of Montague [16] into a two-sorted relational type theory, with possible worlds providing an additional basic type. Although the type theory in [19] validates Extensionality, its language essentially is the language employed here, so that the translation can also serve as a translation into ITL. A minor variation will treat names as they are treated above.

\section{Worlds}

ITL is a generalization of the usual formulation of type theory and intensionality is obtained by giving up the axiom of Extensionality, not by the introduction of possible worlds, as in modal logic. However, while the usual Kripke-style semantics is not known to do a very good job regarding the puzzles of intensionality we have been concerned with here, it does perform very well when it comes to modal reasoning, temporal reasoning, counterfactual reasoning etc. So it seems that worlds and the possibility to quantify over worlds are still welcome, even to those who accept the claim that the present approach to intensionality is superior to the modal one.

If such a combination of modality with true intensionality is desired, one way to proceed would be to simply add domains of worlds to the existing intensional models and interpret a modal higher order language on the results, a course of action followed in Muskens [21. There is, however, an easier way. Once true intensionality is obtained in the way it was done in this paper, worlds can also be constructed out of propositions, the inhabitants of the domain $D_{\langle\rangle}$, while accessibility relations can be obtained as well. The procedure will only be sketched in this section; more formal considerations and comparisons with 
standard approaches to modality will be left to a future occasion.

The idea of constructing possible worlds out of other entities is an old one. E.g. Wittgenstein [33] constructs them out of 'states of affairs' and Carnap 4] takes worlds to be 'state-descriptions', maximal consistent sets of sentences. A recent construction of worlds from propositions can be found in Pollard [23]. Varying upon such proposals, one can identify worlds with certain objects of type $\langle\langle\rangle\rangle$ here, i.e. objects whose extensions are sets of propositions. Here is the construction. Assume, for simplicity, the principles $(\alpha),(\beta)$, and $(\eta)$ discussed above, and let $\Omega$, which will stand for the predicate 'is a world', be a fixed constant of type $\langle\langle\langle\rangle\rangle\rangle$, while $w$ varies over objects of type $\langle\langle\rangle\rangle$. Stipulate the following.

(W1) $\forall w(\Omega w \rightarrow \neg w \perp)$

(W2) $\forall w(\Omega w \rightarrow(w(A \subset B) \leftrightarrow \forall \vec{x}(w(A \vec{x}) \rightarrow w(B \vec{x}))))$

The first of these axioms requires world extensions to be consistent while addition of the second schema makes worlds distribute over logical operators. Statements such as the following become derivable.
a. $\forall w(\Omega w \rightarrow(w(\neg \varphi) \leftrightarrow \neg(w \varphi)))$
b. $\forall w(\Omega w \rightarrow(w(\varphi \wedge \psi) \leftrightarrow((w \varphi) \wedge(w \psi))))$
c. $\forall w(\Omega w \rightarrow(w(\forall x \varphi) \leftrightarrow \forall x(w \varphi)))$
d. $\forall w(\Omega w \rightarrow(w(\exists x \varphi) \leftrightarrow \exists x(w \varphi)))$

The first of these statements says that worlds are complete, while the last two are 'Henkin properties' that enforce, for example, that if an existential proposition is an element of the extension of a given world some proposition witnessing the existential must also be an element. In general, given (W1) and (W2), worlds single out sets of propositions that could be simultaneously true.

It is natural from this perspective to introduce a constant $w_{0}$ of type $\langle\langle\rangle\rangle$ that is meant to denote the actual world, the world consisting of all propositions that are true (in a given intensional model). If this is wanted one should stipulate the following.
a. $\Omega w_{0}$
b. $\forall p_{\langle\rangle}\left(w_{0} p \leftrightarrow p\right)$.

The first of these requirements merely stipulates that $w_{0}$ is a world while the second makes it the actual world. In models additionally satisfying Extensionality, $w_{0}$ is the only world in view of the fact that there are exactly two propositions in such models, but in other intensional models there is no such trivialization. Note, by the way, that nothing requires co-extensional worlds to be identical. The set of propositions that are true in some world does not necessarily determine it. 
Since worlds are of type $\langle\langle\rangle\rangle$ it is possible to iterate and form propositions $w_{1} \varphi, w_{2}\left(w_{1} \varphi\right), w_{3}\left(w_{2}\left(w_{1} \varphi\right)\right)$ and so on. Is it acceptable that these differ in truth value? Here we shall assume that this is not so and that if a proposition $w \varphi$ is true at some world, it is true at all, provided $w$ is a world. The question whether a proposition is in the extension of a world should arguably be worldindependent itself. The way to enforce this is by adopting the following axiom scheme.

(W3) $\forall w w^{\prime}\left(\left(\Omega w \wedge \Omega w^{\prime}\right) \rightarrow\left(w\left(w^{\prime} \varphi\right) \leftrightarrow\left(w^{\prime} \varphi\right)\right)\right)$

In a similar vein, the question whether an $\langle\langle\rangle\rangle$ object is a world, presumably should also be uniform across worlds:

(W4) $\forall w\left(\Omega w \rightarrow \forall w^{\prime}\left(\Omega w^{\prime} \leftrightarrow w\left(\Omega w^{\prime}\right)\right)\right)$

We now have worlds, but we still do not have accessibility relations between worlds. These can be obtained, however, by considering more expressions of type $\langle\langle\langle\rangle\rangle\rangle$. If $R$ is such an expression, it can be interpreted as the predicate 'is accessible', and $\lambda w \lambda w^{\prime} \cdot w\left(R w^{\prime}\right)$ will play the role of an accessibility relation. The usual relational properties (transitivity, reflexivity, euclideanness,...) can then either be stipulated or, depending on the choice of $R$, be shown to hold. For example, the universal accessibility relation $\lambda w \lambda w^{\prime} . w\left(\Omega w^{\prime}\right)$ is easily seen to be an equivalence relation on the set of worlds in view of (W4).

A next step is the introduction of the usual modal operators. Modal boxes can be obtained by writing $[R]$ for $\lambda p \forall w((\Omega w \wedge R w) \rightarrow w p)$, so that $[R] \varphi$ will reduce to $\forall w((\Omega w \wedge R w) \rightarrow w \varphi)$. Diamonds are obtained as usual, as the duals of boxes: $\langle R\rangle$ is short for $\lambda p . \neg[R] \neg p$. Note that if $w^{\prime}$ can be shown to be a world, the statement $w^{\prime}([R] \varphi)$, i.e. $w^{\prime}(\forall w((\Omega w \wedge R w) \rightarrow w \varphi))$, will be equivalent with $\forall w\left(\left(w^{\prime}(\Omega w) \wedge w^{\prime}(R w)\right) \rightarrow w^{\prime}(w \varphi)\right)$ by the distribution of worlds over logical operators and the last statement will in its turn be equivalent with $\forall w\left(\left(\Omega w \wedge w^{\prime}(R w)\right) \rightarrow w \varphi\right)$ by (W3) and (W4).

Let us give another example of an accessibility relation some of whose properties follow from its definition. The relation of belief considered in the previous section, $\lambda p \lambda x$.believe $x p$, is one of explicit belief. It is not closed under entailment or even under logical equivalence. But there is also a notion of implicit belief that is closed under entailment. Roughly, one implicitly believes $\varphi$ if one rationally should believe $\varphi$ given one's explicit beliefs. One way to model this (for some arbitrary agent john) is to consider the following property $R$ of worlds.

$$
\lambda w . \forall p((\text { believe john } p \leftrightarrow w(\text { believe john } p)) \wedge(\text { believe john } p \rightarrow w p))
$$

Here a world $w$ is accessible if John's explicit beliefs in $w$ are exactly those that John actually holds and if those explicit beliefs are in fact true in $w$. There may fail to be such worlds, for example if John's explicit beliefs are in fact inconsistent, a situation not ruled out by our previous considerations. But it is possible to stipulate that $\lambda w \lambda w^{\prime} \cdot w\left(R w^{\prime}\right)$ is in fact serial:

$\forall w \exists w^{\prime} \forall p\left(\left(w(\right.\right.$ believe john $p) \leftrightarrow w^{\prime}($ believe john $\left.p)\right) \wedge\left(w(\right.$ believe john $\left.\left.p) \rightarrow w^{\prime} p\right)\right)$ 
Such a stipulation is simultaneously an existence requirement on worlds and a rationality constraint on John's beliefs. It will lead to the derivability of the usual $\mathbf{D}$ axiom, as $[R] \varphi \rightarrow\langle R\rangle \varphi$ will now hold for all $\varphi$. Note that the definition of $R$ immediately gives transitivity and euclideanity of $\lambda w \lambda w^{\prime} . w\left(R w^{\prime}\right)$, so that we have, for all $\varphi$, that $[R] \varphi \rightarrow[R][R] \varphi$ and $\langle R\rangle \varphi \rightarrow[R]\langle R\rangle \varphi$. These correspond to the usual $\mathbf{4}$ and $\mathbf{5}$ axioms.

\section{Conclusion}

In this paper we have introduced an abstract and simple notion of intensional model that is a generalization of Henkin's general models. Its definition does not involve concepts that have no immediate intuitive justification, such as the "abstraction designation functions" of Fitting [10] or the "application operators" of Benzmüller et al. [3]. These operators provide generalized, non-standard notions of abstraction in one case and of application in the other, but seem to have no justification other than a purely technical one. The present approach, in contrast, gives a kind of minimal logic of intension and extension, with ingredients that well-nigh any logic of intension and extension seems to need. Models are inhabited by intensions, a function $I$ sends terms to their intensions and functions $E_{\alpha}$ send intensions to the extensions they determine. If an additional requirement should be made that the $E_{\alpha}$ be injective, one essentially obtains Henkin's general models, if, moreover, the $E_{\left\langle\alpha_{1} \ldots \alpha_{n}\right\rangle}$ should be required to be onto $\mathcal{P}\left(D_{\alpha_{1}} \times \cdots \times D_{\alpha_{n}}\right)$, standard models are obtained.

The logic contrasts with other approaches to (hyper-)intensionality in two ways. Firstly, unlike other approaches, the aim is not to set up a new logic, but to provide existing classical type theory with a wider class of models in order to invalidate the axiom of Extensionality, which is unwanted in many applications. Secondly, the logic is agnostic about what intensions are. To the latter question various answers have been given but here we have only provided an abstract characterization of the notion of intensionality. We have, in other words, focused on the logic rather than on the ontology of intensions.

While the logic is a generalisation of classical type theory, not an extension with new concepts, it turns out that there is a natural connection with the usual notion of modality. Intensional models may have domains of the propositional type \langle\rangle that are not isomorphic with $\{0,1\}$ and certain properties of objects in these domains can be identified with possible worlds. Accessibility relations of various kinds between such worlds are easily definable and modal box and diamond operators can be obtained accordingly.

\section{Acknowledgements}

I wish to thank Nissim Francez for providing me with detailed comments and for urging me to include linguistic applications. Prof. Roger Hindley very kindly helped me with obtaining a copy of Takahashi's paper on cut-elimination in 
type theory. The anonymous referee's highly welcome comments led to several improvements, among which is the incorporation of possible worlds semantics in section 8 ,

\section{References}

[1] P. B. Andrews. Resolution in Type Theory. Journal of Symbolic Logic, 36(3):414432, 1971.

[2] J. Barwise. Information and Impossibilities. Notre Dame Journal of Formal Logic, 38(4):488-515, 1997.

[3] C. Benzmüller, C. E. Brown, and M. Kohlhase. Higher Order Semantics and Extensionality. Journal of Symbolic Logic, 69, 2004.

[4] R. Carnap. Meaning and Necessity. Chicago UP, Chicago, 1947.

[5] G. Chierchia and R. Turner. Semantics and Property Theory. Linguistics and Philosophy, 11:261-302, 1988.

[6] A. Church. A Formulation of the Simple Theory of Types. Journal of Symbolic Logic, 5:56-68, 1940.

[7] M.J. Cresswell. Intensional Logics and Logical Truth. Journal of Philosophical Logic, 1:2-15, 1972.

[8] M.J. Cresswell. Structured Meanings. MIT Press, Cambridge, MA, 1985.

[9] M. Fitting. First-Order Logic and Automated Theorem Proving. Springer, New York, 1996.

[10] M. Fitting. Types, Tableaus, and Gödels God. Kluwer Academic Publishers, Dordrecht, 2002.

[11] G. Frege. Über Sinn und Bedeutung. In G. Patzig, editor, Funktion, Begriff, Bedeutung. Fünf Logische Studien. Vanden Hoeck, Göttingen, 1892.

[12] L. Henkin. Completeness in the Theory of Types. Journal of Symbolic Logic, 15:81-91, 1950.

[13] J. Hintikka. Impossible Possible Worlds Vindicated. Journal of Philosophical Logic, 4:475-484, 1975.

[14] D. Lewis. General Semantics. In D. Davidson and G. Harman, editors, Semantics of Natural Language, pages 169-218. Reidel, Dordrecht, 1972.

[15] R. Montague. Universal Grammar. Theoria, 36:373-398, 1970. Reprinted in [29].

[16] R. Montague. The Proper Treatment of Quantification in Ordinary English. In J. Hintikka, J. Moravcsik, and P. Suppes, editors, Approaches to Natural Language, pages 221-242. Reidel, Dordrecht, 1973. Reprinted in [29].

[17] Y. Moschovakis. Sense and Denotation as Algorithm and Value. In Logic Colloquium '90 (Helsinki 1990), volume 2 of Lecture Notes in Logic, pages 210-249. Springer, Berlin, 1994.

[18] R.A. Muskens. A Relational Formulation of the Theory of Types. Linguistics and Philosophy, 12:325-346, 1989.

[19] R.A. Muskens. Meaning and Partiality. CSLI, Stanford, 1995. 
[20] R.A. Muskens. Sense and the Computation of Reference. Linguistics and Philosophy, 28(4):473-504, 2005.

[21] R.A. Muskens. Higher Order Modal Logic. In P. Blackburn, J.F.A.K. van Benthem, and F. Wolter, editors, Handbook of Modal Logic, Studies in Logic and Practical Reasoning. Elsevier, Dordrecht, 2006. (to appear).

[22] S. Orey. Model Theory for the Higher Order Predicate Calculus. Transactions of the American Mathematical Society, 92:72-84, 1959.

[23] C. Pollard. Hyperintensional Semantics in A Higher-Order Logic with Definable Subtypes. In Maribel Fernández, Chris Fox, and Shalom Lappin, editors, Lambda Calculus, Type Theory, and Natural Language, pages 32-46, London, 2005. King's College.

[24] D. Prawitz. Hauptsatz for Higher Order Logic. Journal of Symbolic Logic, 33(3):452-457, 1968.

[25] V. Rantala. Quantified Modal Logic: Non-normal Worlds and Propositional Attitudes. Studia Logica, 41:41-65, 1982.

[26] K. Schütte. Syntactical and Semantical Properties of Simple Type Theory. Journal of Symbolic Logic, 25(4):305-326, 1960.

[27] R.M. Smullyan. First-Order Logic. Springer-Verlag, Berlin, 1968.

[28] M. Takahashi. A Proof of Cut-elimination Theorem in Simple Type Theory. Journal of the Mathematical Society of Japan, 19(4):399-410, 1967.

[29] R. Thomason, editor. Formal Philosophy, Selected Papers of Richard Montague. Yale University Press, 1974.

[30] R. Thomason. A Model Theory for Propositional Attitudes. Linguistics and Philosophy, 4:47-70, 1980.

[31] R. Turner. A Theory of Properties. Journal of Symbolic Logic, 52(2):455-472, 1987.

[32] A. N. Whitehead and B. Russell. Principia Mathematica. Cambridge University Press, 1910-13.

[33] Ludwig Wittgenstein. Tractatus Logico-Philosophicus. Routledge, 1922.

[34] E. Zalta. A Classically-Based Theory of Impossible Worlds. Notre Dame Journal of Formal Logic, 38(4):640-660, 1997. 